\title{
Impronta del acosmismo luriánico en la Ethica de Spinoza ${ }^{1}$
}

\author{
(Footprints of Lurianic Acosmism \\ on Spinoza's Ethica)
}

Miquel BeLTRÁN

Recibido: 12 de marzo de 2014

Aceptado: 27 de abril de 2015

\section{Resumen}

El objetivo del artículo es exponer la doctrina acosmista que se halla en la cábala de Luria, tal como la describe Abraham Cohen de Herrera en Puerta del cielo, y cómo pudo ocurrir que a través de la lectura de esta obra aquella doctrina influyera en la concepción de Spinoza sobre la irrealidad de los modos.

Palabras clave: cábala luriánica, Infinito, Spinoza, modos, privación.

\begin{abstract}
The aim of this paper is to explain the doctrine of acosmism, as found in the Kabbalah of Luria, in the way it is described by Abraham Cohen de Herrera in his treatise, Puerta del cielo, and to postulate that the influence of this work could lead Spinoza to his conception on the unreality of modes.
\end{abstract}

Keywords: Lurianic Kabbalah, Infinite, Spinoza, Modes, Privation.

En un artículo revelador para el entendimiento del Dios spinoziano, Mukhopadhyaya se refería a la interpretación formalista de los atributos "de acuer-

\footnotetext{
${ }^{1}$ La investigación previa a la redacción de este artículo ha sido posible gracias a la concesión a su autor de una International Fellowship in Jewish Studies de la Memorial Foundation for Jewish Culture de Nueva York 2014-2015.
} 
do con la cual un atributo es solo una subjetiva adscripción de cualidad a la substancia que carece de ellas" 2 , y observaba que un adicional apoyo a esta descripción descansa en el dictum spinoziano según el cual toda determinación es negación ${ }^{3}$. Proseguía: "Puesto que toda determinación es negación, la substancia solo puede ser definida negativamente, y los atributos, aun siendo infinitos en número, habrán de ser considerados meramente subjetivos; la substancia infinita permanece indiferente a tales adscripciones subjetivas ${ }^{4}$ ". La definición de substancia, en el libro primero de la Ethica, daría soporte asimismo a la interpretación subjetivista, puesto que "si la substancia es aquello que existe en sí mismo y es capaz de concebirse por sí mismo, nuestra concepción de aquella en términos de los infinitos atributos debe necesariamente ser contemplada como meramente subjetiva e ideal" 5 . Determinatio negatio est ${ }^{6}$ comporta que Spinoza es reacio a admitir cualquier caracterización positiva de la substancia en tanto que infinita -o considerada en sí misma-, y le aboca a la teología negativa, pese a que, de modo explícito, la Ethica parece demostrar que todo puede ser afirmado de Dios. Esta aparente paradoja nos lleva a poder contemplar el realismo y racionalismo spinozianos como un constructo a la medida de nuestro entendimiento, pues en el fondo el pensador es escéptico sobre la capacidad de la razón para comprender aquello que es en sí. Además, "si nos adherimos a esta versión, la atribución de acosmismo resulta ineludible. Solo Dios o la substancia habrán de admitirse como trascedentes frente a todas las determinaciones y las relaciones. El mundo con su multiplicidad no puede ser considerado como una manifestación o emanación real de Dios, sino solo como una apariencia ilusoria"7.

\footnotetext{
2 Mukhopadhyaya (1950-51) p. 180.

3 Cf. Melamed (2012), pp. 175-196.

${ }^{4}$ Mukhopadhyaya, p. 180.

5 Mukhopadhyaya, p. 180.

6 Esta es la expresión tal como se halla en la epístola 50 de Spinoza: "En cuanto a que la figura es una negación, y no algo positivo, está claro que la materia, considerada de forma indefinida, no puede tener ninguna figura, y que la figura tan solo se halla en los cuerpos finitos y determinados. Pues quien dice que percibe una figura, no indica otra cosa sino que concibe una cosa determinada y cómo está determinada. Por tanto, esta determinación no pertenece a la cosa según su ser, sino que, por el contrario, es su no ser. De ahí que, como la figura no es sino una determinación, y la determinación es una negación (et determinatio negatio est), no podrá ser, según se ha dicho, otra cosa que una negación". Los textos de Spinoza, tras la primera referencia a la obra, en la que se escribirá el título completo, se citarán según la convención internacional. Siglas de las obras: KV para Korte Verhandeling, TTP para Tractatus Theologico-Politicus, E para Ethica ordine geometrico demonstrata, Ep para Epistolae. En cada una de las citas se detallará lo siguiente: tras la abreviatura del título y la ubicación de la cita en la edición canónica de Gebhardt (Spinoza Opera 1924, reedición de 1972)-tomo, número(s) de página(s) y número(s) de línea(s)-, indicamos la página en las traducciones castellanas utilizadas, a saber: la de Vidal Peña para la Ethica, y las de Atilano Domínguez para el resto de obras, todas publicadas en Alianza Editorial. Así, esta primera cita es Ep50, SO 4, 240, 6-15. AE 1305, 309 (esto es, Epístola 50, tomo cuarto de Spinoza Opera (reedición de 1972), página 240, líneas 6 a 15. Spinoza, Correspondencia, Alianza Editorial número 1305, página 309.

${ }^{7}$ Mukhopadhyaya, pp. 180-181.
} 
Spinoza parece querer eludir, con todo, ciertas incongruencias en las que están condenados a caer los cabalistas, como asimismo la tradición neoplatónica en general. Por ello habría decidido adherir en lo posible el atributo a la substancia, en un intento de evitar la problemática y terminología emanatistas (que sin embargo perduran en los modos infinitos). Como Verman observa, "los cabalistas pugnaron por hacer mella en la dicotomía entre la transcendencia divina y la inmanencia, y formularon una concepción radical de la deidad que combina ambos principios. Afirmaban que Dios en sí mismo se halla dividido en dos aspectos distintos"8. Hallamos en la cábala de Luria9 que En Sof es y no es idéntico al mundo, siendo lo que procede de él, a la vez, su negación. A través de esta paradoja es como el lenguaje tiende a aproximarse a la naturaleza del Infinito. Pero pensamos que Spinoza emprendió la inverosímil tarea no solo de querer exponer lo anterior en terminología filosófica, sino también de demostrarlo geométricamente. En el sistema luriánico, siendo el tsimtsum - el encogimiento de Dios en sí mismo que permite la aparición de un espacio vacío en el que emanaciones de su luz darán origen al mundoun acto, a la vez, de ocultamiento y de revelación, puede decirse que el Infinito se contrae en el lenguaje, y de modo específico en la combinación de letras que comprende las así llamadas 'diez formulaciones de la creación'. Esta contracción es, a la vez, el esconderse y el manifestarse de la esencia de Dios ${ }^{10}$.

En Sof, en uno de sus momentos liminares, es la unidad indiferenciada y primigenia previa a la concentración de su esencia que se produce a través del tsimtsum y del lenguaje. Así, una de las razones para que se produzca el tsimtsum, en Puerta del Cielo, el texto cabalístico escrito en castellano por Abraham Cohen de Herrera en Ámsterdam, entre 1620 y 1632, y a través del cual pudo Spinoza adentrarse en los pormenores de la doctrina de Luria, es la siguiente: "Relatándose a sí misma a

\footnotetext{
8 Verman, (1987), pp. 25-37. La cita es de la página 28.

9 Rabí Isaac de Luria, Ari (el león) (1534-1572) asquenazí, nació en Jerusalén. Huérfano desde los ocho años, su familia había recalado en Egipto, donde él estudió en la yesivá de R. David ibn Zimra, el mayor halajista de su tiempo. Luria encontró un mercader que poseía un libro esotérico, probablemente una copia del Zóhar, que había sido impreso por primera vez en aquellos años. Quiso comprárselo, pero el hombre replicó que se lo regalaría si Luria convencía a su tío, cobrador de impuestos, que le liberara de ciertas cargas fiscales. Una vez dueño del libro, Luria se recluyó y dedicó al estudio del texto. Seis años después, empezó a tener sueños en los que una voz le urgía a abandonar Egipto, diciéndole que el discípulo al que debía enseñar su doctrina estaba en Safed, y se llamaba Hayyim. Luria pasó sus últimos años en esta ciudad, en la que se estableció entre 1569 y 1570. Introdujo innovaciones en la cábala por las que impulsó una nueva concepción de la misma, en aspectos relativos al origen de la emanación, como la del tsimtsum, encogimiento o repliegue de Dios en sí mismo -aunque el concepto se halla ya en el Talmud-. También resulta novedosa la doctrina de la rotura de los vasos, y la de la restauración de las caras.

10 Cfr. Dunn (2008), p. 29: "El uno divino se exilia a sí mismo para rozar a sus criaturas exiliadas. Isaac Luria y sus discípulos entendieron la inefable conexión entre Creador y creado... como una contienda espiritual que se desenvuelve hacia la mutua reabsorción de los unos en el otro".
} 
otros, no según su infinidad, a nadie comunicable ni de ninguno participable, sino según aquella finita porción o parte que pueden participar los efectos, unos más y otros menos, y todos limitadamente [...] Y este es el tsimtsum o encogimiento [.....] que el Rab con translación y corporal metáfora considera en la Causa Primera, para dar razón de la producción de sus finitos efectos"11. Y más adelante: "La segunda manera, con que podemos decir que en algún modo se estrecha y limita en sí la infinita Causa primera, para producir fuera de sí finitos y limitados efectos, es por su entendimiento y voluntad, con que conociéndose y queriéndose a sí misma, no según su imparticipable inmensidad, que apartada de todo en sí puramente consiste, sino según aquella porción y deficiente imitación que de ella puede participar el efecto; se refiere a él relatando su única infinidad, por varios y limitados modos, a varios y limitados futuros efectos que con su voluntad produce; concluyendo que determinando su indeterminada esencia a determinados modos de comunicación, que fuera de sí conoce y quiere, hace tsimtsum, y casi encoge y estrecha su infinita potencia, para producir finitos efectos"12. Esta doctrina del tsimtsum, la retractación o encogimiento, trata de dar respuesta a la cuestión de cómo es posible que el mundo exista y que no haya nada allende el Infinito o En Sof. Luria elude la complejidad de soluciones anteriores y propone una concepción diferente en su sentido íntimo. La cábala de Girona y otras corrientes influidas por el neoplatonismo aducían que Dios proyectó su poder creativo más allá de sí mismo -algo inconcebible si lo penetra y alcanza todo en su infinitud-. De su esencia oculta emanan, según la jerarquía ontológica de aquella doctrina, las sefirot o luces divinas, que son lugares en los que Dios se manifiesta a sí mismo y que contienen los arquetipos de todas las cosas que existen. El progreso de la emanación divina procede hacia lo bajo de tal modo que deviene, paulatinamente, menos espiritual; así llegan a constituirse los mundos, al materializarse la luz, que en un inicio era sublime e inabarcable.

En la cábala luriánica de Herrera leemos, inversamente, que los producidos son, a la vez, su misma Causa: "de manera que podemos decir que todos los producidos y cada uno de ellos, son, o es, su improducida Causa, así porque lo que comunicó a otros contiene en sí misma, como porque todo lo que contiene en sí es su misma

11 Cohen de Herrera, Abraham, Puerta del cielo, f. 95 r y f 95 v. Se cita por el manuscrito Koninklijke Bibliotheek, 's-Gravehage 131 C 10 (manuscrito de la Biblioteca Real de la Haya, copiado de mano anónima, completo en 10 libros, con sumario e índice de materias). Existen otros tres manuscritos, dos de ellos conservados en la Biblioteca Ets Haim-Montezinos de la sinagoga portuguesa en Ámsterdam (48 A 16, copiado por Samuel David Curiel en 1675, completo) y HS 48 B 19 (copiado por Samuel Abaz George en 1740, al que le faltan los libros octavo, noveno, y décimo). Un cuarto manuscrito, también incompleto (le faltan asimismo los tres últimos libros), y escrito por copista anónimo, se halla en la biblioteca de la Columbia University de Nueva York (x 86 H 42 Q). Las referencias se dan por el número de folio, recto y verso. La ortografía se ha actualizado según las normas admitidas por el Grupo de Investigación Siglo de Oro (GRISO) de la Universidad de Navarra.

12 Puerta del cielo, f. 97 r. 
simplicísima infinidad y existencia; mas no son dicha su Causa en su ilimitada e improducida eminencia, a nadie comunicable, sino en su propia finita y causada naturaleza, porque todo lo que de ser y perfección en sí participan, es de su causa y por su causa, y todo lo que de no ser y falta en sí incluyen, es por la degeneración y caída con que se alejan de su Causa y se llegan al nada. De modo que como la Causa Primera es todas las cosas que son o pueden ser [.....] porque cada una, y todas, carecen y son privadas de la infinita perfección primera, así porque son limitadas y contraídas a determinada 13 naturaleza, como porque proceden de otro, sin lo cual no son ni se conservan, ni pueden obrar ni obran, ni conseguir el fin pretendido"14. Nótese que la terminología spinoziana de la producción y del obrar se halla ya en este texto de Herrera, como asimismo la consideración de las cosas como modos. Herrera sentencia: "Con que se puede concluir, que como la Causa Primera es todas las cosas en modo incausado e infinito, así las mismas cosas que de ella en todo dependen, son dicha causa, mas en modo causado y finito, y como dice nuestro preceptor y maestro, en tsimtsum o encogimiento, mediante el cual comunicó En Sof la Causa Primera, fuera de sí, mas no apartado de sí, lo que era y tenía en sí, mas no del modo que era y lo tenía en sí" 15 .

La cábala de Luria y de ciertos de sus discípulos considera los errores de la formulación emanatista -abogando por la no-separación entre lo producido y la Causa Primera-. Por lo demás, la procesión había devenido, en cabalistas anteriores, cada vez más compleja, y se dio una dialéctica entre la luz directa que desciende de Dios, y la luz refleja, que torna a él, lo que introdujo un elemento de tensión en aquellas concepciones. Luria, en su intento de eludir la paradoja ínsita en la doctrina de la emanación, introduce la doctrina del tsimtsum, según la cual el primer acto del Infinito no es su revelación a algo otro, y, lejos de ser una procesión fuera de Dios, es su retraimiento en Sí mismo. Mediante esta acción, Ensof abandona una región de sí al vacío, dando espacio para los mundos que van a ser. Dios puede, así, manifestarse, solo porque primero se contrae en Sí mismo. Pero las interpretaciones de esta contracción pueden conducir a una nueva paradoja: una lectura literal del relato luriánico comporta que la esencia divina se halla por completo ausente del espacio en el que se despliega el aparato de la creación. Pero también podrá argüirse que la divina esencia, pese a retraerse, permanece presente -en su función de Causa Primera-, en el dominio de lo creado.

Se consideró el tsimtsum, por parte de los primeros -quienes abogaban por la literalidad-, como un mito acerca de la creación, que pretende solventar las inconsistencias que forman parte del despliegue gradual de las emanaciones, pero algu-

\footnotetext{
13 'Terminada' en el manuscrito.

14 Puerta del cielo, f. 94 v.

15 Puerta del cielo, f. 94 v. Es pues en el modo en lo que lo producido difiere de la causa, pero no en su entidad.
} 
nos cabalistas que siguieron la estela de Luria lo consideraron una metáfora de la historia de Dios, una categoría funcional que afirma y subvierte, a la vez, la trascendencia radical. Dios como En Sof existe paralelamente a Dios como finito (la infusión de Dios en el espacio vacío creado por su propia contracción). Se reconoce así que el proceso de inmanencia comporta una transformación en la vida de Dios. El primero que articuló esta interpretación no-literal fue, precisamente, Herrera, quien debido a su obcecado afán por conciliar la doctrina de Luria con la argumentación filosófica, pensó de Dios que era la potencia última que sustenta los contingentes en el mundo creado, y que, por la misma razón, no podría estar ausente del espacio que había causado en sí mismo, sino que todo lo penetraba, de modo que cada una de las criaturas depende de Él, en el sentido de que está en Él. El retraimiento del tsimtsum es, de este modo, parangonable a una ocultación. La no-contingencia del infinito último hace que se extienda a todas las categorías del ser, que sin embargo no difiere de él. En la cábala, esta dualidad que no es tal se halla por primera vez explícitamente formulada en la obra de Herrera. Una razón para lo anterior es que tanto este como Yosef Ergas ${ }^{16}$ tendieron a conciliar el sistema de Luria no solo con el neoplatonismo, sino también con las enseñanzas de Cordovero ${ }^{17}$, quien por su lado tampoco había eludido recurrir a la argumentación filosófica cuando ésta podía servir para clarificar nociones y desarrollos cabalísticos. Asimismo Israel Saruq ${ }^{18}$, el maestro de Herrera, quien inició a este en el conocimiento de las doctrinas luriánicas, había añadido expresiones a la descripción del tsimtsum, del tipo 'como si fuera' 19 , que aparecen también en Puerta del Cielo, donde además el tsimtsum se define como metafórico en iterados pasajes. Así, ya Saruq sugería una lectura noliteral del encogimiento. Las interpretaciones del Zóhar que hizo Luria tendían a la

\footnotetext{
16 Rabí Yosef Ergas, nacido en Liorna (1685-1730), escribió el Séfer Shomer Emunim (Guardián de los fieles), texto publicado en 1737, y en el que se expone uno de los más refinados argumentos en favor de la interpretación metafórica del tsimtsum, oponiéndose así a quienes lo consideraban una contracción divina en sentido literal.

17 Moisés ben Jacob Cordovero (1522-1570) fue el cabalista que lideró la escuela de Safed en el siglo XVI, y probablemente Luria fue su discípulo. Herrera cita a Cordovero en múltiples ocasiones, en concordancia con la preeminencia que le otorga frente a los otros cabalistas a los que se refiere Herrera en Puerta del cielo, una predilección que no es ajena al particular uso que Cordovero hace de la filosofía en el esclarecimiento de la naturaleza del Infinito y de sus emanaciones.

18 Israel Saruq (c. 1560-1610) propaló en ciertos lugares de Europa manuscritos que contenían las enseñanzas de Isaac de Luria, de quien se proclamaba discípulo. En Puerta del Cielo Herrera refiere la observación que le hizo el mismo a Saruq en Ragusa, la actual Dubrovnik, de modo que, tal como se arguye, pudo ser en esta ciudad donde se conocieran, y donde el último le aleccionó sobre los misterios de la cábala en el peculiar modo que permitiría a Herrera perpetrar el sincretismo entre esta y el neoplatonismo del que hace gala en sus escritos. León de Módena, gran rabino de Venecia, le profesó una proverbial antipatía, y acusó a Saruq de poner en práctica técnicas teúrgicas y mágicas para procurarse ventajas sociales en la ciudad de los canales. Cf. a este respecto Dweck (2011:136-141).

19 Herrera gusta de utilizar la expresión 'casi', en sentido idéntico al que conforma esta expresión de Saruq, como una consideración aproximativa, diríamos pseudo-ontológica.
} 
abstracción ${ }^{20}$. El elemento metafórico es perceptible ya en su propia formulación, y tanto él como los discípulos que copiaron sus discursos afirmaron que las imágenes míticas no deben ser tomadas literalmente, sino en una correspondencia alegórica.

De acuerdo con la novedosa interpretación que hace Luria del concepto rabínico de tsimtsum ${ }^{21}$, En Sof deja un espacio para la creación contrayéndose de modo que pueda llenar dicho espacio con las emanaciones de su luz. La creación toma forma a partir de estas emanaciones de un incomprehensible Infinito, que carece de distinciones intrínsecas, y cuya luz fluye para crear el cosmos. Pero esta luz es reflexiva, y vuelve a Él. Así, la existencia del mundo consiste en un proceso sustentado y metafórico de contracción divina, cuyas emanaciones retornarán al infinito. Se sugiere que lo múltiple es aquella última realidad distorsionada, fragmentada como en infinitos espejos 22 .

El acosmismo luriánico se despliega en la doctrina según la cual Dios se concreta como esencia a través de las centellas de su luz que informan todas las cosas, pero que son Él mismo 23 . Lo anterior halla expresión, terminológicamente, en la distinción entre tsimtsum ke-peshuto (tsimtsum literal) y tsimtsum ke-eyno peshuto (tsimtsum metafórico), y persistió entre los discípulos de Luria. Pero los literalistas tuvieron que enfrentarse a comentarios zoháricos como "ningún lugar está vacío de É124" y se vieron forzados a admitir que aunque la providencia de Dios se extiende

20 En Casa de la divinidad se expone con mayor detenimiento la doctrina de las quelipot o cáscaras (también, etimológicamente, escorias) mediante la cual en la cábala luriánica se explica la naturaleza y origen del mal.

21 Tal y como Novak probó en un magistral trabajo, la noción se halla ya en los escritos rabínicos. Novak halla la primera plasmación de la misma en un midrás en torno a la revelación, considerada esta una relación que comporta el dar y recibir activamente: "Ves que cuando el Sagrado [....] dio la Torá a Israel, si hubiese venido a ellos con su entera potencia, (ellos) habrían sido incapaces de soportarla, como se dice en la Escritura: "Estas palabras habló Jehová a toda vuestra congregación en el monte, de en medio del fuego, de la nube y de la oscuridad, a gran voz; y no añadió más" (Dt 5,22). Así, Él vino a ellos de acuerdo con su fuerza, como se dice en la Escritura: "Voz de Jehová con potencia; Voz de Jehová con gloria" (Sal 29, 4). No dice "con su potencia" (be-kokho), sino "con potencia" (bakoah): de acuerdo con la fuerza de cada uno de ellos". Novak concluye: "En la Torá, la incapacidad de Israel para soportar la voz de Dios en su entera fuerza se trata de resolver al proponer a Moisés como intermediario, y consiguiendo que Dios acepte el compromiso (Dt 5, 24-25). Pero en el midrás, Dios milita su propio poder. Mientras que la Escritura invoca un intermediario externo a Dios, el midrás percibe el auto-confinamiento reflexivo de Dios. El midrás desarrolla la idea como sigue: "Cuando el Santo [....] dijo a Moisés, "Y harán un santuario para mí, y habitaré en medio de ellos" (Ex 25,8), él se sorprendió y dijo: "La gloria del sagrado llena los dominios celestes y terrenales, y aún así dice "Y harán un santuario para mí, y habitaré en medio de ellos". Y el sagrado dijo: "Yo no pienso como tú piensas [.....] pero descenderé y contraeré (ve'azamzem). Mi presencia (shekinati) a las dimensiones del santuario" (1992: 301). Se trata de Shemot Rabá 34:1.

22 La metáfora se halla también en textos cabalísticos, como el Libro de los espejos. Cfr. Matt (1980), pp. 129-172. La recurrencia de la metáfora en la cábala la prefiguran ciertos versículos bíblicos.

23 Cfr. Fine (2003).

24 Zóhar 3.257b. 
a todo, su presencia no está en todo. Los no-literalistas, sin embargo, tienen que habérselas con el problema que introduce el que Dios se halle en todas partes, aun en los resultados de la creación más abyectos y execrables, algo que Spinoza admite en una carta a Blyenbergh, en la que sentencia que "el matricidio de Nerón....en cuanto incluía algo positivo, no era un crimen: pues también Orestes realizó la acción externa y tuvo además la intención de asesinar a su madre y, sin embargo, no es acusado tanto como Nerón. ¿Cuál fue entonces el crimen de Nerón? Simplemente que con dicha acción él demostró que era ingrato, inmisericorde, y desobediente. Ahora bien, es cierto que nada de esto expresa esencia y que, por tanto, tampoco Dios fue causa de ello, aunque haya sido causa del acto y de la intención de Nerón" 25.

Habrá que señalar que la doctrina luriánica permite que el panenteísmo y el acosmismo no sean doctrinas antagónicas, sino que puedan darse a la vez. Podrá aducirse, además, que En Sof es ein (nada), y que lo mejor que puede hacerse a su respecto es sumirse en el silencio. Pero es misión de la humanidad, a la vez que reconoce las distinciones en el seno de lo múltiple, captar la unidad de todas las cosas. En Puerta del cielo Herrera sostiene la no-entidad de los efectos emanados, de esta suerte: "Ya se probó, y es cierto, que todos los efectos imitan y se asemejan a la universal Causa Primera, y que su ser no es otro que una participación de su eficaz principio26". Son los efectos "el casi limitarse y multiplicarse con el tsimtsum o encogimiento, que el sapientísimo Luria metafóricamente introduce, no en sí, que siempre fue, es, y será el mismo invariable y libre de toda limitación y movimiento, sino en hábito 27 a sus efectos"28. No hay multiplicidad para el ilimitado En Sof. Y ni siquiera hay uno, propiamente hablando: "La Causa Primera no es uno, principio de número, porque este se reduce a la cantidad discreta o apartada, y es accidente; ni uno corporal, que es propio de la cantidad continua, y que aunque individido es, en sus partes, infinitamente divisible; no es tampoco uno esencial, que aunque trascendente, es determinado a esta o aquella naturaleza, como un ser, una vida, un entendimiento, etc... que no es primero, puro y absoluto uno, sino tal o tal uno, postrero, dependiente, compuesto, infeccionado, imperfecto, menesteroso y decaído"29. El primer efecto, en Herrera, aunque causa de las causas, no es causa de sí mismo, sino que es causado. Y por el contrario, "enseña R. Simeon ben Iohay ${ }^{30}$, de feliz memoria, (la distinción) que hay entre la Causa de todas las causas y la Causa

\footnotetext{
25 Ep23, SO 4, 147, 8-16. AE 1305, 210-211.

26 Puerta del cielo, f. 95 v.

27 'Habitud' en el manuscrito.

28 Puerta del cielo, f. 95 v.

29 Puerta del cielo, f. 30 r.

30 Como se hallaba establecido en su tiempo, Herrera creía que Rabí Simeón ben Yohay, un tanna del siglo II d. C, había sido el autor del Zóhar.
} 
de las causas, de que la primera es sin duda el incausado Causador de todo, Ensof el sobrealabado, y la segunda, a lo que entiendo, la mahassabá o mente alta [...] y en resolución, el primer y perfectísimo efecto de la independiente causa de todas las causas, y que en su virtud, y con su concurso, es causa de las siguientes e inferiores causas, mas no de todas las causas, como Ensof el inefable, porque a serlo fuera causa de sí mismo, que aunque causa de causas, es también causado"31. O también: "Todo ser es contenido y está en el ser primero, y que lo es por esencia, y como tal es absoluto, puro, e ilimitado ser en sí, y por sí subsistente, de modo que todo ser, fuera de este primero e ilimitado ser, es casi parte, porción, y deliberación suya, siendo tanto más o menos perfecto cuanto privado más o menos de él" 32 . Es de observar que Herrera no parece diferenciar aquí entre privación y negación en tanto que tales nociones, como lo hará Spinoza en una carta a Blyenbergh en la que nos detendremos más adelante. Pero el cabalista sí distingue, mediante esta terminología, entre dos suertes de infinito: "Hay infinito privativo e infinito negativo. El privativo es aquel que, conviniéndole tener fin, no lo tiene, y el negativo es el que no conviniéndole tener fin, no lo tiene; que por otros términos, mas con la misma intención y sentencia, se llaman infinito que carece o es privado de fin, e infinito que excluye todo fin o término33". Y prosigue: "de estos dos infinitos, el primero, excluyente, fin, y negativo, es En Sof, la Causa Primera, y el segundo, careciente de fin y privativo, es la materia primera, y la naturaleza o esencia de cada cosa en potencia a su ser y existencia, virtud, operación, hábito, y último fin y cumplimiento; y del primero procede y nace el segundo 34 ".

Así pues, dos suertes de infinito, uno de una previedad sublime, excluyente, que "se sublima y separa de todas las demás cosas, sin que tenga con ninguna de ellas, ni con todas, ninguna proporción ni conveniencia [...] de manera que podemos decir que se remota, aparta, y casi huye de todas las cosas, aquella absoluta y exenta sublimidad y eminencia que es del todo separada y libre de todo término y fin [...] mas en cuanto la dicha infinidad divina se relata a sus futuros efectos, por varios modos de comunicación que les puede participar, nacen las existencias y formas que limitan, y actualmente perfeccionan a la indefinida materia y no perfecta esencia, que llamamos infinito privativo y carente de término, dependiente del infinito negativo y que excluye todo término"35. Concluye Herrera: "con que podemos decir, que en cuanto excede a todo, (la Causa Primera) hace tsimtsum y se recoge en sí, como aquella que casi retirada en el abismo de su infinidad, es incomparablemente de todas apartada, y en cuanto se comunica a todos, es totalísimamente causa de todos,

\footnotetext{
31 Puerta del cielo, f. 36 v.

32 Puerta del cielo, f. $97 \mathrm{v}$.

33 Puerta del cielo, f. 99 r.

34 Puerta del cielo, f. 99 v.

35 Puerta del cielo, f. 99 v.
} 
y en todos todo, como si a sí misma se infundiera en todos e hiciera a todos, como por la infusión de su luz, que de ella no se distingue"36.

Mukhopadhyaya dio en ver, en su capital artículo, que esta distinción en el infinito se da también en Spinoza: "La concepción tripartita de la sustancia -substancia como el uno absoluto ${ }^{37}$, substancia como la eterna causa de todo, y la substancia como en efecto todo-, que incide sobre la doctrina spinoziana de los atributos y de los modos, ha dado lugar a ciertas dificultades, tanto teóricas como prácticas. Se cuestiona: Si la substancia debe concebirse por sí y existe por sí, ¿cómo habrá que entender su concepción como la causa de todas las cosas, y como la totalidad de todo lo que existe?"38, para recordarnos que "de estas tres concepciones, la primera es la definición de la substancia con que la Ethica se inicia39". Así, la definición de substancia estaría por el absoluto que se halla más allá de la natura naturans y la natura naturata, asociada esta última terminología a las dos respectivas concepciones de la substancia, que se dan tan solo "a la luz de la doctrina de los atributos y de los modos 40". La natura naturans es Dios en cuanto se lo considera causa libre -en la terminología de Herrera, el infinito privativo- tal como se lo define en E1P29S: "Antes de seguir adelante, quiero explicar aquí -o más bien advertir- qué debe entenderse por naturaleza naturante, y qué por naturaleza naturada. Pues creo que consta, por lo anteriormente dicho, que por naturaleza naturante debemos entender lo que es en sí y se concibe por sí, o sea, los atributos de la substancia que expresan una esencia eterna e infinita, esto es [...] Dios, en cuanto considerado como causa libre 41 ". Así, resulta claro que natura naturans no refiere la substancia en tanto que substancia, sino como causa libre de aquello que se sigue de su naturaleza, esto es, si nos atenemos a la terminología del TTP, a "Dios en relación con las cosas creadas 42 ". Se trata del Dios cuya esencia es idéntica a su poder de pro-

\footnotetext{
36 Puerta del cielo, f. $100 \mathrm{v}$.

37 Cf. Ariew (1990), pp. 16-31.

38 Mukhopadhyaya, p. 179.

39 Mukhopadhyaya, p. 179.

40 Mukhopadhyaya, p. 179.

41 E1P29S, SO 2, 71, 5-12. AE H 4404, 96. (Esto es, Ethica ordine geometrico demonstrata, Libro primero, proposición 29, escolio, en Spinoza Opera, tomo segundo, página 71, líneas 5 a 12. Traducción en Alianza Editorial, número H 4404, página 96). Cf. Melamed (2013), en un intento de refutación de Curley (1969). Remite Melamed asimismo a su anterior trabajo, cf. Melamed (2010b), pp. 128-142. 42 Leemos: "Hay que señalar además, que, aparte de Jehová, no se halla en la Escritura ningún nombre que indique la esencia absoluta de Dios, sin relación a las cosas creadas. Y por eso los hebreos defienden que solo este es el nombre propio de Dios, mientras que los demás son simples apelativos.
} 
ducir las cosas, y así también, con la voluntad y el entendimiento, pues es un Dios dotado de esencia, a diferencia de la substancia en tanto que infinita, vastísima previedad a la esencia. En Herrera, la distinción entre dos infinitos se concreta también en otros términos: "el que se llamó arriba alto de los altos no es el alto sobre todos los altos; y este misterio no se manifestó a todos los sabios y profetas" 43 . De este modo, "la primera unidad precede al primer ente, que consta y es compuesto de ser y esencia, así el primer esencial y originario número antecede a la multitud de todos los entes y producidas cosas44". En Spinoza, si Dios en sí mismo es la substancia más allá de la propia natura naturans, esta última sería la Primera Causa que en Herrera es la actividad más universal, la causa eficiente de todas las cosas que se comunica a todo. Herrera la llama en cierta ocasión, anticipando a Spinoza, "la más que sustancia, unicísima, sencillísima, e ilimitada Causa Primera"45. Puesto que el cabalista admite que las divinas lumbres o sefirot son sustancias diversas, a la unicidad divina la considera como más que estas en su sustancialidad. También: "sustancial sujeto o supuesto de quien por emanación resultaron"46 las visibles especies, propiedades, y potencias ${ }^{47}$. Escribe también que todo emana de su sustancia, quedando sin embargo, ella, como "su receptáculo, sujeto, y vaso"48. De la otra descripción de la anterioridad de Dios respecto de su propia esencia en la Ethica, el infinito en cuanto infinito, hallamos una prefiguración en el siguiente pasaje de Puerta del cielo: "Pero siendo la Causa Primera verdaderamente infinita, como en otra parte se ha probado, es menester que para casi determinarse y limitarse en sí, haya alguna como diferencia y apartamiento de sí, saliendo (según el modo de nuestro entendimiento) casi por movimiento, de sí a otro fuera de sí (aunque realmente en sí misma, y lo mismo que ella misma), y en resolución, de infinito a finito, $[\ldots . .$.$] no el infinito en cuanto infinito, sino una finita, casi porción suya, resulta$ el metafórico movimiento con que el ilimitado parece que se limita, y saliendo de sí a otros, produce de su infinidad finitos efectos" 49 . Herrera sostiene que el primer y más perfecto efecto solo difiere de la causa en que es un efecto: "con que se concluye que hay sumo efecto a quien el Sumo Bien se ha sumamente comunicado, y que es el mayor y mejor que puede ser producido, y tan excelente en el orden de los

\footnotetext{
La verdad es que los otros nombres de Dios, sean sustantivos o adjetivos, son atributos que convienen a Dios, en cuanto es considerado en relación a las cosas creadas, o se manifiesta a través de ellas." (TTP13, SO 3, 169, 3-13. AE 1185, 302). Cf. Beltrán (1995) p. 27ss.

43 Cf. Puerta del cielo, f. 103 r.

44 Puerta del cielo, f. 6 r y f. 6v.

45 Puerta del cielo, f. $90 \mathrm{v}$.

46 Puerta del cielo, f. 90 v.

47 Cf. La definición de sustancia en Cohen de Herrera (2002), p. 180: "sustancia es aquel ente, que teniendo propio ser, no se arrima o sustenta en otro, antes es en él en quien los accidentes inhieren, estriban, y posan; o la sustancia es aquel ente que no ha menester sujeto ni arrimo". (p. 180).

48 Puerta del cielo, $90 \mathrm{v}$.

49 Puerta del cielo, f. 95 r y 95 v.
} 
efectos, como lo es su Causa, entre todas las causas, de la cual solamente difiere en ser efecto 50 ".

En Spinoza, la esencia de las cosas no puede concebirse sin Dios, pero la substancia se concibe en sí y por sí, y por tanto, al margen de las esencias y existencias de los modos que produce, separada también del Dios creador que está en relación con las cosas que se siguen necesariamente de su naturaleza. Sabemos que "de la necesidad de la naturaleza divina deben seguirse infinitas cosas de infinitos modos (esto es, todo lo que puede caer bajo un entendimiento infinito) 51 ". Es en extremo importante entender que el intelecto infinito que se refiere aquí es un modo, esto es, una manifestación divina. Dios produce algo que permite probar, ya que este modo infiere un número de propiedades que se siguen de la definición de Aquél, que de Él deben seguirse infinitas cosas de infinitos modos. Esta demostración, que parte de cómo Dios es entendido por el entendimiento a partir de su definición, creemos que confirma la interpretación acosmista, puesto que la razón de que se sigan infinitas cosas de Dios es que una de entre estas entiende que todas deben seguirse de la necesidad de su naturaleza, y por tanto, la multiplicidad existe solo del lado de los modos. Porque toda determinación es negación, todo lo que define al modo, menos aquello que lo conserva en la existencia, que es la existencia misma en la que es ${ }^{52}$, lo niega al mismo tiempo. Cabe aquí introducir la definición de negación tal como se expone en las cartas a Blyenbergh, en el largo fragmento en el que se diferencia entre privación y negación, de modo diverso, sea dicho en passant, a como Herrera postulaba la distinción entre infinitos: "sigo adelante, explicando los términos negación y privación, e indicando brevemente cuanto es necesario para descubrir con más claridad el sentido de mi carta anterior.

Digo, pues, en primer lugar, que la privación no es el acto de privar, sino tan solo la pura y simple carencia, que por sí misma no es nada [...] solo es un ente de razón o modo de pensar que formamos al comparar unas cosas con otras. Decimos, por ejemplo, que un ciego está privado de la vista porque lo imaginamos fácilmente como vidente, ya surja esta imaginación de compararlo a él con otros que ven, o de comparar su estado actual con el anterior, en que veía. Cuando consideramos así a ese hombre, comparando su naturaleza con la de otros o con la suya pasada, afirmamos que la vista pertenece a su naturaleza, y por eso decimos que ahora está privado de ella. Pero cuando se considera el decreto de Dios y su naturaleza, no tenemos más razón para decir que ese hombre está privado de la vista que lo está la piedra, ya que en ese momento es tan contradictorio atribuir la vista a ese hombre como a la piedra. Porque a ese hombre no le pertenece como propio más que lo que le atri-

\footnotetext{
50 Puerta del cielo, f. 40 v.

51 E1P16. Las infinitas cosas de infinitos modos son solo todo lo que puede caer bajo un entendimiento infinito, es decir, todo lo que conoce un modo infinito.

52 De aquí la centralidad del conatus.
} 
buye el entendimiento y la voluntad de Dios. Por consiguiente, Dios no es más causa del no ver de aquel que del no ver de la piedra, la cual es una simple negación.

Asi también, cuando consideramos la naturaleza del hombre que es guiado por el apetito de placer y comparamos su apetito actual con el que tienen los justos, o con el que tuvo en otro tiempo él mismo, decimos que ese hombre está privado de un apetito mejor, porque pensamos que le pertenece el apetito de la virtud. Pero no podemos hacer lo mismo si atendemos a la naturaleza del decreto y del entendimiento de Dios, ya que, en ese caso, dicho apetito no pertenece actualmente a la naturaleza de ese hombre más que a la naturaleza del diablo o de la piedra. Y por eso, en este sentido, el apetito mejor no es privación, sino negación. En una palabra, la privación no es más que negar de una cosa algo que juzgamos que pertenece a su naturaleza, y la negación no es más que negar de una cosa algo que no pertenece a su naturaleza 53 ". Pero si la negación es negar de una cosa algo porque no pertenece a su naturaleza, y toda determinación es negación, las determinaciones no pertenecen a la naturaleza de aquello que determinan, y por lo tanto, los modos no existen sino en cuanto puras manifestaciones de la existencia, al margen de lo que los determina para nosotros en forma de individualización. No que sean ilusorios desde nuestra perspectiva, sino que las determinaciones modales no son, objetivamente, sino negaciones ${ }^{54}$. Cualquier determinación de la substancia debe ser considerada como una derivación en forma de non-entia. Tan a ultranza existe solo Dios que es negación la pluralidad de los modos, cuya realidad como manifestaciones de la substancia es solo verdadera para el entendimiento infinito, este último también otra determinación. Es a partir de una negación como las cosas se siguen necesariamente del poder de Dios, pues Él se produce solo a Sí mismo, en un sentido que Herrera, siguiendo a Luria, pudo prefigurar para Spinoza, pues "en el primer efecto produjo la Causa Primera a todos los efectos, y que concurriendo con él y con todos los demás, a todas sus operaciones y efectos, es más causa de ellas y de ellos, que ellos mismos, y que pudiendo producir todo por sí y sin medios, los quiso producir y produjo por ellos, y esto a fin de comunicarse, más y más perfectamente, a sus efectos, y ser más gloriosa y cognoscible en ellos" ${ }^{55}$. Los diferentes aspectos del universo, esto es, la configuración de lo múltiple, son solo desde la perspectiva del ser creado. En términos de En Sof, en su esencia, todo es uno, y no hay otro que infinito. Las divisiones y niveles del ser pueden darse sin haber multiplicidad en él: "Porque yo, Yavé, no me he mudado"56. Y sin embargo hay un significado para la manifestación de Dios, y es realizar la revelación de su perfección, como también

\footnotetext{
53 Ep21, SO 4, 128, 835, 129, 1-6. AE 1305, 194-195. Cfr. Sobre esta cuestión el artículo de Demos (1933), 155-166.

54 Cf. Aquila, (1978) 271-288.

55 Puerta del cielo, f. 61 v. Se trata de una variación de la primera proposición del Liber de Causis. $56 \mathrm{Mal}$ 3, 6.
} 
el propiciar el amor de los modos hacia Él, que será el propio amor de Dios por Sí mismo, y para el cual precisa causarse a sí mismo como esencia, y además, a todo lo que como causa libre dicha esencia puede producir. Por ello es tan importante para Spinoza que Dios haga todo lo que hace, como vindica frente a sus adversarios con inusitada vehemencia en E1: "Otros piensan que Dios es causa libre porque puede, según creen, hacer que no ocurran -o sea, que no sean producidas por Élaquellas cosas que hemos dicho que se siguen de su naturaleza, esto es, que están en su potestad. Pero esto es lo mismo que si dijesen que Dios puede hacer que de la naturaleza del triángulo no se siga que sus tres ángulos valen dos rectos, o que dada una causa, no se siga de ella un efecto, lo cual es absurdo" 57.

El propósito de la contracción, en Luria, es que Dios configure una esencia en Sí mismo. Pero no para que haya algo distinto de Él, pues nada se da fuera de Él. Melamed 58 ha argüido, en lo reciente, que si los modos se contemplan como ilusorios, la cuestión de la razón por la cual emanan de la única substancia o son producidos, resulta problemática. Sostiene Melamed que no podríamos llegar tampoco al tercer género de conocimiento a través de una idea adecuada de la esencia de las cosas singulares si los modos no fuesen reales, y más importante, no cabría aducir que el orden y conexión de las cosas en todos los atributos es el mismo, como Spinoza hace en E2P7S59, pues no se daría pluralidad en dicho mundo. Aduce el estudioso que Spinoza sostuvo que adquirimos un mayor conocimiento de Dios cuanto más conocemos las cosas naturales, pero esto es afirmado también a la inversa, tal y como en un pasaje de TTP queda claro: "como nada puede ser ni ser concebido sin Dios, es cierto que cuantas cosas existen en la naturaleza, implican y expresan el concepto de Dios en proporción a su esencia y a su perfección60". Este pasaje parece correr paralelo a ciertas proposiciones del libro último de la Ethica, como E5P25D: "El tercer género de conocimiento progresa, a partir de la idea adecuada de ciertos atributos de Dios, hacia el conocimiento adecuado de la esencia de las cosas. Cuanto más entendemos las cosas de este modo, tanto más entendemos a Dios y, por ende, la suprema virtud del alma, esto es, su potencia o naturaleza suprema [...] consiste en conocer las cosas según el tercer género de conocimiento". Además, el conocimiento natural puede ser llamado divino, "puesto que nos es dictado, por así decirlo, por la naturaleza de Dios, en cuanto que participamos de ella, y por sus decretos" 61 . Y más adelante: "Dado, pues, que nuestra alma, por el simple hecho de que contiene objetivamente, en sí misma, la naturaleza de Dios, y par-

\footnotetext{
57 E1P17S, SO 2, 61, 27-32, 62, 1. AE H 4404, 80-81. Cf. a tal respecto Carriero (1995), pp. 245-273. 58 Melamed (2010a), p. 77-92.

59 "Un modo de la extensión y la idea de dicho modo son una y la misma cosa, expresada de dos maneras" (E2P7S, SO 2, 90, 8-9, H 4404, 130-131).

60 TTP4, SO 3, 59, 32-35, 60, 1-8. AE 1185, 138.

61 TTP1, SO 3, 16, 10-14. AE 1185, 76.
} 
ticipa de ella, tiene poder para formar ciertas nociones que explican la naturaleza de las cosas $[. .$.$] con razón podemos afirmar que la naturaleza del alma [...] es la pri-$ mera causa de la divina revelación62". Así, según Spinoza, entendemos las esencias de las cosas adecuadamente a partir de la idea adecuada de ciertos atributos de Dios. Pero esta consiste en que aquellos son expresiones subjetivas, en el entendimiento, de lo que constituye la esencia divina, esto es, expresiones en el entendimiento de aspectos de la existencia infinita que es Dios. El tercer género de conocimiento, contra lo que Melamed parecen concluir, no tiene su origen en el conocimiento de las cosas y de sus esencias, sino en la idea adecuada de los atributos 63 , los cuales se identifican además, en E2, con su esencia formal. No hallamos en la Ethica que la idea adecuada de los modos obligue a concluir que estos tengan existencia en sí mismos. Iteradamente se nos recuerda que son en otro y concebidos por otro, de manera que su idea adecuada es que consisten en manifestaciones de la única substancia, y así se los considera según el tercer género de conocimiento. De nuevo, el entendimiento infinito es parte de la naturaleza, un modo en sí mismo, y conoce aquello que contiene la totalidad de la naturaleza desde la perspectiva del modo, tal como leemos en Ep32: "Por lo que respecta al alma humana [...] considero que es una parte de la naturaleza, y la razón es que yo afirmo que en la naturaleza se da también un poder infinito de pensar, el cual, en cuanto es infinito, constituye en sí objetivamente toda la naturaleza, y sus pensamientos proceden del mismo modo que la naturaleza, es decir, que su objeto (ideatum). Afirmo [...] que el alma humana es ese mismo poder, no en cuanto que es infinito y percibe toda la naturaleza, sino en cuanto que es finito, es decir, en cuanto solo percibe el cuerpo humano, y en este sentido afirmo que el alma humana es una parte de cierto entendimiento infinito"64. El poder del intelecto infinito es aprehender la totalidad de la naturaleza, no el Infinito en sí. Nuevamente, un pasaje de Puerta del Cielo resulta definitivo sobre esta cuestión, el siguiente: "Siendo la intención del infinito y primer agente producir las criaturas y comunicarse y manifestarse a ellas, y consiguiendo este fin no por las superiores sefirot o numeraciones, que como muy cercanas a su fuente, quedaron improporcionadas a lo bajo y encubiertas, sino por las inferiores, y en especial por la décima y más baja, que es maljut 65 , en las cuales y en la cual se proporciona y acomoda (lo que no hace en las más altas) a la capacidad de los creados mundos, y de los abstractos, angélicos, y humanos entendimientos" 66 . En Sof, así, se acomo-

62 TTP1, SO 3, 15, 25-28. AE 1185, 75.

63 Martens (1978), pp. 107-111,

64 Ep32, SO 4, 173, 16-19, 174, 1-6. AE 1305, 238. Cfr. sobre esta carta, y la concepción spinoziana de los todos y las partes, Sacksteder (1977), pp. 139-159.

65 Maljut es el nombre de la décima sefirá, llamada también 'cohabitación divina', e identificada con la shejiná o presencia divina. Cf. Montag (2002), pp. 131-144 sobre el influjo de la noción en Spinoza. También en lo reciente Huenemann (2014) discurre sobre la posibilidad de esta vinculación.

66 Puerta del cielo, f. 8 v. 
da al modo de conocer tanto de la mente abstracta como de las humanas, que estarían por el entendimiento infinito y finito en Spinoza.

Es una petición de principio concebir como ilusorio el mundo porque se opone a una caracterización objetiva del mismo que no puede darse, según los presupuestos. Siguiendo a Cosenza, pensamos que "la exigencia acosmista prescinde [...] del mundo del finito, y afirma que la única realidad concebible es la del ser infinito. Acosmismo, así, quiere decir prioridad del absoluto de Dios, en el orden del conocimiento y en el orden de la naturaleza"67. No cabe plantearse que los modos son ilusorios, con la carga de significado que esta adjetivación comporta. El conocimiento adecuado de los mismos convierte, sin embargo, en ilusorio aquello por lo cual unos modos son distintos de otros. Los estudiosos rechazan el acosmismo sobre esta base, ignorando que los modos -si nos referimos a ellos por lo que los diferencia- carecen de existencia real en sí mismos. La existencia en sí de sus determinaciones es incierta. El conocimiento adecuado que tenemos de ellos es, por lo demás, parte del entendimiento infinito de Dios, que -como referíamos- es, él mismo, un modo, así que nada de esto está vinculado con Dios en tanto que infinito. En este sentido, Herrera pretendió acercarse al conocimiento de Aquel hasta donde fuese posible: "siendo nuestra intención alcanzar de la Causa Primera lo más que nos es posible, es necesario que para subir a tan sublime perfección y eminencia, apartemos y removamos del conocimiento de ella, por negación, todas las imperfecciones y determinadas perfecciones, que comparadas a la infinita, como carecientes de ella, son en algún modo imperfectas"68.

Dios se conoce a Sí mismo cuando su entendimiento, ya sea infinito o finito, conoce las cosas en lo que tienen en común con él, esto es, en cuanto manifestaciones de la eterna existencia que es Dios. Spinoza escribe en el libro V de la Ethica: "Concebimos las cosas como actuales de dos maneras: o bien en cuanto concebimos que existen con relación a un tiempo y lugar determinados, o bien en cuanto concebimos que están contenidas en Dios y se siguen unas de otras en virtud de la necesidad de la naturaleza divina [...] las que se conciben como verdaderas o reales de esta segunda manera, las concebimos desde la perspectiva de la eternidad, y sus ideas implican la eterna e infinita esencia de Dios" 69 . Los modos considerados según esta segunda vía carecen de determinaciones, y por la misma razón, estas son irreales, y pertenecen a una concepción inadecuada de las cosas.

Finalmente, Melamed arguye que el idealismo es una doctrina que no puede ser mantenida por Spinoza, puesto que él sostiene que los atributos son conceptualmente (y causalmente) independientes entre sí, por E1P10: "Cada atributo de una misma

\footnotetext{
67 Cosenza (1953) p. 193.

68 Puerta del cielo, f. 68 r.

69 E5P29S, SO 2, 298, 30-32, 299, 1-3. AE H 4404, 447-448.
} 
substancia debe concebirse por sí"70, y E2P6D: "En efecto, cada atributo se concibe por sí, prescindiendo de cualquier otro"71.

Si Spinoza no reduce la extensión al pensamiento, ni a la inversa, ambos se hallan con todo subsumidos en aquello único cuya esencia expresan (o constituyen para el entendimiento): la existencia infinita ${ }^{72}$. Si el idealismo significa que la extensión se reduce al pensamiento, Spinoza no es idealista. Si significa que tanto la extensión como el pensamiento se reducen a expresiones, para un entendimiento que no es en sí ni se concibe por sí, de la existencia, entonces sí sería idealista, porque ni la extensión ni el pensamiento son otra cosa que concepciones de un entendimiento que, ya sea infinito y o finito, es un modo. Los atributos se conciben en sí y son en sí, tales los percibe el entendimiento. Melamed considera que la separación conceptual de los atributos es inconsistente con el monismo, pero haciendo esto, interpreta la filosofía de Spinoza desde la perspectiva que el propio orden de exposición de la Ethica parece querer eludir. Se parte del entendimiento, que en efecto concibe cada uno de los atributos en sí. Pero sabemos que, en Dios, los atributos no comportan diversificación conceptual: la extensión es existencia, al igual que el pensamiento, y ambos constituyen la esencia divina solo para el entendimiento. Desde la perspectiva de la existencia, nada los diferencia.

Verman señalaba "la inviabilidad del acosmismo como un principio de religión. Aunque el panenteísmo puede ser fundamentalmente paradójico, es propicio a conducir, no obstante, a la religiosidad, pues Dios se halla ínsito en todos los aspectos de la existencia. En consecuencia, el judaísmo tradicional, cuando despliega la ley religiosa [.... en cada fase de la vida, podía beneficiarse de tal doctrina. El acosmismo, por su parte, es potencialmente subversivo, pues niega el valor intrínseco de cualquier cosa salvo de Dios. Aunque los místicos [...] puedan dar respuesta al nihilismo que esto comporta, este es simplemente demasiado radical para el vulgo en general73". Acaso por ello Spinoza se declara abiertamente panenteísta: "Todo cuanto es, es en Dios, y sin Dios nada puede ser ni concebirse"74. Pero oculta la previedad divina a la constitución de su propia esencia, esto es, a su auto-existenciación, a la que solo alude cuando se refiere a la substancia como es en sí misma, o a la substancia en tanto que infinita, pero que se halla en la consideración literal de la causa sui, y también en la parte quinta de la Ethica, por la que comprendemos que

\footnotetext{
70 E1P10, SO 2, 51, 28-29. AE H 4404, 66.

71 E2P6D, SO 2, 89, 8. AE H 4404, 128.

72 Cf. Gilead (1985), pp. 72-78. Cf. también Gilead (1990), pp. 454-475. Huenemann supone que la conclusión lógica de la consideración del vínculo entre el intelecto infinito y la mente humana aboca a Spinoza al acosmismo. Leemos: "es innegable que el sistema conduce de modo dramático hacia el acosmismo" (p. 106).

73 Verman, Mark, pp. 34-35.

74 E1P15, SO 2, 56, 24-25. AE H 4404, 73.
} 
Dios se configura en esencia que causa o produce las cosas que puede producir -aunque en Él mismo- para alcanzar la otredad que le permitirá, a través del amor intelectual de los hombres hacia Él, amarse a Sí mismo. Se trata pues, de una procesión ética -como el mismo título del libro indica-, no ontológica. Acorde, además, con la prioridad de la primera sobre la última, que es constante en la tradición hebrea.

\section{Referencias bibliográficas}

Aquila, R. (1978): "The Identity of Thought and Object in Spinoza", Journal of the History of Philosophy 16, pp. 271-288.

ArIEw, R. (1990): “The Infinite in Spinoza's Philosophy", en E. Curley y P.-F. Moreau (eds.), Spinoza: Issues and Directions. Leiden, Brill, pp. 16-31.

Beltrán, M. (1995): "The God of the Tractatus Theologico-Politicus", North American Spinoza Society Monographs 3, pp. 23-33.

CARriero, J. (1995): "On the Relationship between Mode and Substance in Spinoza's Metaphysics", Journal of the History of Philosophy 33, pp. 245-273. Cohen de Herrera, A. (sin fecha), Puerta del cielo. Koninklijke Bibliotheek, 'sGravehage 131 C 10. La Haya.

Cohen de Herrera, Abraham (2000): Epitome y compendio de la lógica o dialéctica, ed. por Giuseppa Saccaro del Bugga. Bolonia, Cooperativa Libraria Universitaria Editrice Bolonia.

CosenzA, P. (1953): “Acosmismo e platonismo in Spinoza", Annali della facoltà di lettere e filosofia della Università di Napoli 3, pp. 169-194.

Curley, E. (1969): Spinoza. An Essay in Interpretation. Cambridge, MA, Harvard University Press.

Demos, R. (1933): “Spinoza`s Doctrine of Privation”, Philosophy 8, pp. 155-166.

DunN, J.D. (2008): Window of the Soul. The Kabbalah of Rabbi Isaac Luria. San Francisco, Weiser Books.

Dweck, Y. (2011): The Scandal of Kabbalah. Leon Modena, Jewish Mysticism, Early Modern Venice. Princeton, Princeton University Press.

Fine, L. (2003): Physician of the Soul, Healer of the Cosmos. Isaac Luria and his Kabbalistic Fellowship. Stanford, Stanford University Press.

Gilead, A. (1985): “'The Order and Connection of Things': Are They Constructed

Mathematically-Deductively according to Spinoza?", Kant-Studien 76, pp. $72-$ 78.

Gilead, A. (1990): “Spinoza's Two Causal Chains", Kant-Studien 81, pp. 454-475. Huenemann, C. (2014): Spinoza's Radical Theology. The Metaphysics of the Infinite. Durham, Acumen Publishing Limited. 
MARTEnS, S. C.: "Spinoza on Attributes", Synthese 37, pp. 107-111.

MatT, D. (1980): "David ben Yehuda he-Hasid and his Book of Mirrors", Hebrew Union College Annual 51, pp. 129-172.

Melamed, Y.Y. (2010a): "Acosmism of Weak Individuals? Hegel, Spinoza, and the Reality of the Finite", Journal of the History of Philosophy 48, pp. 77-92.

Melamed, Y.Y. (2010b): "The Metaphysics of the Theological-Political Treatise", en Y.Y. Melamed y M. A. Rosenthal (eds.), Spinoza's Theological-Political Treatise. A Critical Guide. Cambridge, Cambridge University Press, pp. 128142.

Melamed, Y.Y. (2012): “'Omnis determination est negatio'. Determination, Negation, and Self-Negation in Spinoza, Kant, and Hegel", en E. Förster y Y. Y. Melamed (eds.), Spinoza and German Idealism. Cambridge, UK, Cambridge University Press, pp. 175-196.

Melamed, Y.Y. (2013): Spinoza's Metaphysics. Substance and Thought. Oxford, Oxford University Press.

MontaG, W. (2002): "'That Hebrew Word': Spinoza and the Concept of the Shekhinah", en H. M. Ravven y L. E. Goodman (eds.): Jewish Themes in Spinoza's Philosophy. Albany, State University of New York Press, pp. 131-144. Mukhopadhyaya, A. (1950-51): "Is Spinoza an Acosmist?", The Philosophical Quarterly; an Organ of the Indian Institute of Philosophy and the Indian Philosophical Congress, 23, pp. 177.

NovAK, D. (1992): "Self-Contraction of the Godhead in Kabbalistic Theology", en L. E. Goodman (ed.), Neoplatonism and Jewish Thought. Albany, State University of New York Press, pp. 299-318.

SACKsteder, W. (1977): "Spinoza on Part and Whole: The Worm's Eye View", Southwestern Journal of Philosophy 8, pp. 139-159.

SpINoZA, B. (1972): Spinoza Opera. Heidelberg. Carl Winter Universitätsverlag. Primera edición de 1925.

Verman, M. (1987): "Panentheism and Acosmism in the Kabbalah", Studia Mystica 10, pp. 25-37.

Miquel Beltrán

Instituto de Estudios Hispánicos en la Modernidad

Universidad de las Islas Baleares

yobcn9@hotmail.com 